\title{
Trade-off between System Effectiveness and Context Constraints in the Design of an IoT System Giving Access to Health Care in African Rural Villages
}

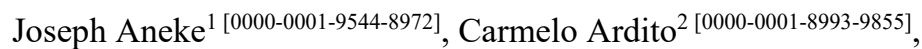

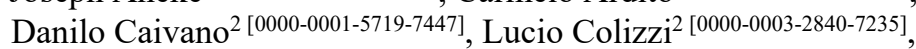 \\ Loredana Verardi ${ }^{2}$ [0000-0002-6833-2840] \\ ${ }^{1}$ ICT/Innovation Department, University of Nigeria \\ - 410001 - Nsukka, Enugu State, Nigeria \\ joseph.aneke@unn.edu.ng \\ ${ }^{2}$ Dipartimento di Informatica, Università degli Studi di Bari Aldo Moro \\ Via Orabona, 4 - 70125 - Bari, Italy \\ \{name.surname\}@uniba.it
}

\begin{abstract}
Aging population is a global concern, but the situation is even worst in developing countries where the migration of the active population from rural areas to major cities makes the elderly left in their home in the village, usually assisted by close relatives who take care of them. Far distance to accessing good health facilities provided in the city hospital usually results to the untimely death of people suffering from treatable and manageable diseases like diabetes, malaria, high blood pressure and waterborne diseases. In this paper, we present the design concept of a system for preventing and controlling people's health conditions; it exploits the possibilities of the Internet of Things technology, still trying to find a convenient balance between effectiveness, reliability and context limitations that require a cheap, affordable and easily configurable system.
\end{abstract}

Keywords: Ambient assisted living, Ubiquitous healthcare, Smart devices, Tailoring by end user.

\section{Introduction}

Supporting elderly or disabled people in their everyday lives has been a longstanding research area. Many researchers have addressed smart aging services, which can help people age comfortably and independently while staying in their current residence as long as possible. Aging population has been a global concern which makes a large impact on government spending and economic policy in healthcare, pension, and social benefits program more critical especially in developing countries. In Africa, a significant percentage of elderly people face distinctive challenges such as chronic diseases including heart disease, physical disabilities, diabetes, and depression [1]. Many of them live in rural village, where it is common for parents to expect their children to be 
around when they are aged or retired from active service. Due to migration and changes in family values, such a culture is becoming unpopular. The elderly are then transferred to nursing homes or remain independent in their home in the village, where they are usually left with close relatives (informal caregivers) who take care of them. Such informal caregivers are often young and technical savvy people.

Unfortunately, assessing good health facilities remains a major concern in rural villages. Very often functional health centers are concentrated in the cities, which are many kilometers far from the villages where professional health givers (doctors) are not available. All of this further worsens the plight of those having health challenges and living in the village, especially the elderly.

A multidimensional approach to integrate diverse healthcare systems are needed to prevent and control health problems of elderly people [2]. Most developed systems focus on the quality of functional aspects of life assistance services that emphasized convenience and effectiveness based on the concepts of hospitalization [1]. These services are usually not affordable, timely and sometimes complex for developing countries like Nigeria, where the annual income per capita is about $\$ 2.100$, with low growth in terms of technological and infrastructure development.

We are currently working on designing a system aiming at improving health of elderly living in rural villages. We are exploiting the Internet of Things (IoT) technology, which offers plenty of possibilities for addressing the issues highlighted above. Because of the limitations of the particular context, the ultimate challenge is the identification of a cheap, affordable and easily configurable system that can be successfully applied and definitely adopted in the considered scenario. The system consists of a heterogeneous combination of apps and Arduino-based devices that connect patients and healthcare service providers remotely located. An important feature from the interaction point of view is that the system is easily configurable by non-technical people, e.g., caregivers.

In this paper that reports on our ongoing work, after a short survey of the related literature (Section 2), we describe the requirements emerged (Section 3) and the solutions proposed in the design-concept (Section 4). Conclusions are finally provided.

\section{$2 \quad$ Related Work}

Advances in sensing and network technologies allow physical objects in everyday lives to be connected with each other and thus data could be shared and work together in a collaborative way. This technological trend, has been influencing applications in many domains, including smart industry [3], smart home [4, 5], smart office [6], smart farm [7], smart grid [8], smart city [9], but also Cultural Heritage [10,11]. Also, it would have a great impact on wellness systems and health-related services deployed in agingin-place solutions. Embedded systems and wireless sensor networks enable a variety of sensors to be incorporated into office buildings, or wearable devices to be attached to occupants and biological signals to be directly collected from their body [12]. Data sets collected from sensors could be utilized to infer situations and help family members or caregivers monitor or perform emergency activities for the elderly and disabled. Dishman highlighted four promising areas (focused on by Intel's Proactive health research 
group) where technologies for adaptive aging could help older adults live healthier and more productive lives: promoting healthy behaviors, early disease detection, improved treatment compliance, support for informal caregiving using embedded systems [13].

Allowing the elderly to live at home with a reasonable level of assistance, called aging in place, is an active research area, and in particular many developed countries have drawn much interest to the related research projects. Ubiquitous healthcare (uHealth) systems are a key to handle the challenges of aging population and growing healthcare cost around the world. Such systems contribute to the revolution in medical history by providing an instant overlook into the diseases and by giving patients an emergency alert in case of any chronic problem [14]. They also enable the service providers/practitioners to remotely monitor the patient's physiological data in real-time and provide feedback [15]. In a u-Health system, sensors (either on-body or implantable) like ECG, EEG, EMG, motions/positioning, and body temperature forward their data via wireless interface to a base station or access point. This data is then streamed to the hospital via a wide coverage network such as 3G, WLAN, LAN or GPRS/SMS to a medical doctor for remote monitoring, or to hospital database for records or telemedicine or to ambulatory service in case of emergency [16]. An off-the-shelf u-Health system is, for example, the "MySignals SW BLE Complete Kit" (see Fig. 1), a development platform for medical devices and eHealth applications to develop personalised eHealth web services ${ }^{1}$. The kit includes 17 sensors which can be used to monitor more than 20 biometric parameters. Further sensors, possibly provided by the user himself, can be added to build new medical devices. All the data gathered by MySignals is encrypted and sent to the user's private account at Libelium Cloud through WiFi or Bluetooth. The data can be visualized in a tablet or smart phone with Android or iPhone Apps. Unfortunately, even if the proposed solution is worthwhile, the cost is as much as $\$ 2000$, which makes it not affordable for the context we are considering.

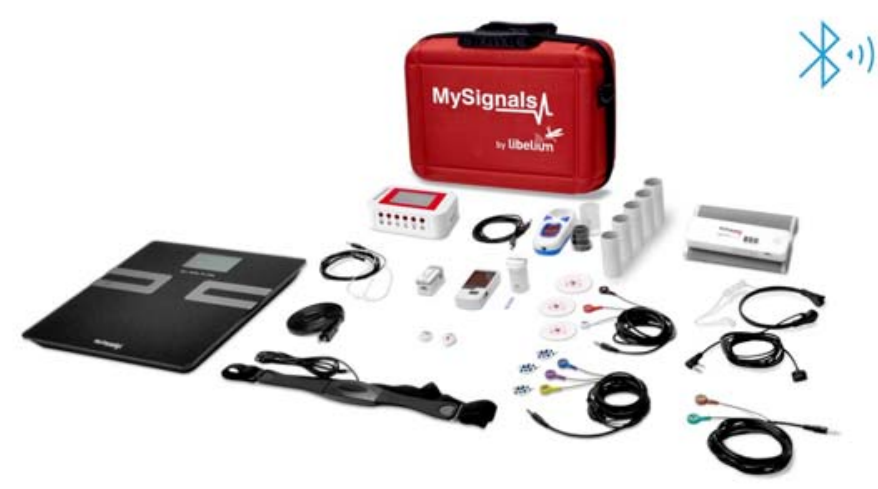

Fig. 1. The MySignals SW BLE Complete Kit (image courtesy of www.cooking-hacks.com).

\footnotetext{
${ }^{1}$ http://www.my-signals.com/
} 


\section{Context and System Requirements}

The primary challenge of a system that has to support the elderly's healthcare in rural villages is that it has to be cheap and easy to use by caregivers who have limited knowledge of technology.

We set-up a design team that included electronic engineers, as well as software engineers and interaction designers. They are the authors of this paper. Most of them are expert with IoT technologies. Moreover, the first author is a PhD student at a Nigerian University, graduated in Electronic Engineering, and currently in our Department for a 5 -month visit. $\mathrm{He}$ is the reference person for illustrating the different facets of the Nigerian reality and providing more insights about the context requirements. Other team members have previous experience in designing technology for Ambient Assisted Living (AAL). See, for example, $[17,18]$.

The system design consisted of three main phases, as described in the following. In order to get more ideas on the system to be envisioned, we also took into account the projects developed as assignment to the PhD students attending a course on "IoT Technologies and Tools for Configuring Smart Environments". The assignment consisted of designing an AAL system for elderly people in destitute areas.

During the first phase, the team identified the context elements that influence the design of the proposed solution. First, the infrastructure limitations were considered and then the stakeholders and their relationships, skills and roles were identified. It emerged that the solution should be cheap and affordable, easy and simple to use with very minimal setup procedures required. It should not rely on highly sophisticated mobile devices since it is intended for rural villages where Internet services may not be available. It must be flexible so that to be adapted to a variety of diseases, stakeholders, devices and infrastructures available.

As depicted in Fig. 2, the elderly person is provided with a device able to detect different parameters (e.g., heart rate, temperature, ECG, blood pressure, glucose, pulse); the collected data are locally stored and later transferred to the mobile device of an informal caregiver, typically wife/husband, sons/daughters, or a relative. The elderly person can use his/her device also for sending SOS alarms to the mobile phone of the informal caregiver. The app here installed allows the caregiver to visualize health parameters and to transfer them to doctors or other professional caregivers. The doctor, using a Web or a mobile app, can access data that have been previously collected from the patient's device and later transferred by the informal caregiver app.

On the basis of the results of the first phase, during the second phase, the team was engaged in designing the device to be provided to the elderly people. After evaluating and discussing different prototypes, it was finally decided that the best design would be a briefcase, or bag, composed of a central unit plus different modules, each able to perform specific measurements, to be added or removed according to the patient's diseases. The name chosen for the system was iHealthBag. The communication modalities among the system components were defined.

Finally, inspired by [17], the design team focused on designing the pill dispenser, a further component of the patient's device that manages drug therapy administration. The pill dispenser is characterized by the following features: 
- modularity, since there are the therapy may change over time;

- accurate reporting of drug intake, so that the caregivers can check that the patient is taking the pills correctly;

- remote therapy modification: if the professional caregiver decides to change the therapy, he/she can remotely program the behavior of the pill dispenser by using the visual interface available on the app installed in his/her computer or mobile phone. It is responsibility of the informal caregiver (usually a close relative of the patient) to change the pills in the dispenser accordingly.

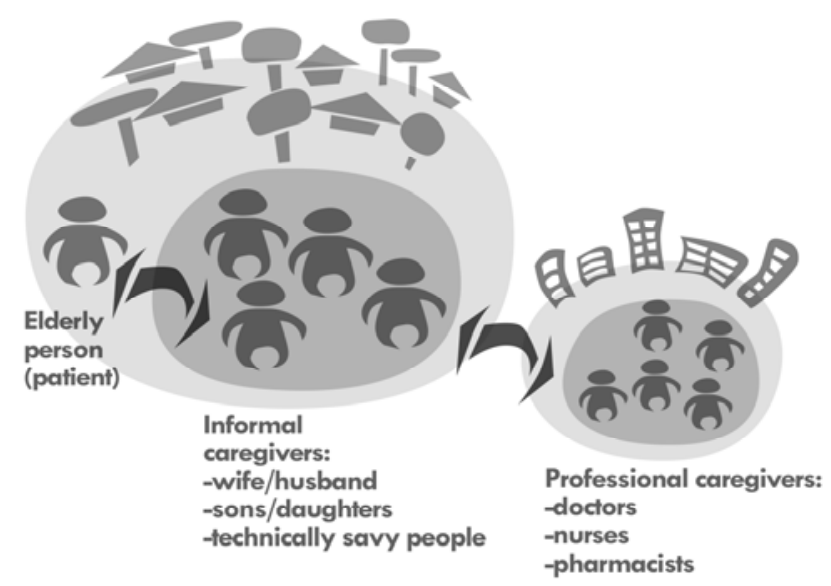

Fig. 2. Stakeholders involved in the African rural village scenario and communications among them.

\section{The iHealthBag}

Because of the limitations of the particular application context, the ultimate challenge has been the identification of a cheap, affordable and easily configurable system that can be successfully applied and definitely adopted. The iHealthBag solution is proposed as a heterogeneous combination of apps and affordable Arduino-based devices that connect patients (elderly) and healthcare service providers remotely. The system deploys a wide variety of sensors and actuator to monitor and observe the patient's vital health signs and interactively connects him/her with the informal caregiver or doctor on 24 hours and 7 days a week basis.

The iHealthBag is composed of a Central Unit device that comes with expandable slots where different combination of sensors, called Sensor Modules, can be inserted (see Fig. 3). The Central Unit contains an Arduino Uno microcontroller board ${ }^{2}$, a Bluetooth module, a nodeMCU board ${ }^{3}$, an SD card shield. Each Sensor Module is a box which provides all the sensors useful for a disease. Several Sensor Modules can be connected, thus several illnesses are monitored. Each box is printed with a $3 \mathrm{D}$ printer

${ }^{2} \mathrm{https}: / /$ www.arduino.cc/

${ }^{3} \mathrm{http}: / /$ nodemcu.com/ 
and contains: one nodeMCU board, the specific sensors and the necessary shields for them, input/output ports if necessary (for example, a jack). The box has also connectors to take and to provide power from/to the boxes it is attached to. The nodeMCU boards available in both the Central Unit and the Sensor Modules permits the data transfer among every component of the iHealthBag.

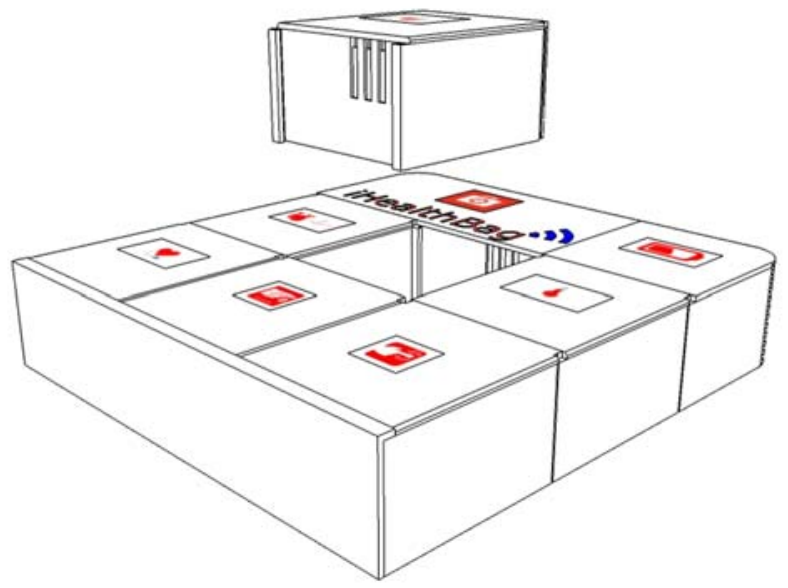

Fig. 3. The iHealthBag patient's device.

The Pill Dispenser Module has the same dimensions of the Central Unit Module. It contains a nodeMCU microcontroller and a number of small boxes that contain pills of different types. Each pill box has a LED light, which is automatically switched on when that pill has to be taken. To confirm that he took the pill, the patient pushes a button on the pill box and the light is switched off.

Data collected by sensors can be stored and retrieved on the SD card available in the Central Unit device and then automatically transferred to Bluetooth recipient, i.e., the informal caregiver's smartphone. Optionally, the iHealthBag can be provided with a GSM module: in this case, as a Sensor Module receives input of an anomaly with the system, the iHealthBag immediately sends message alerts to the caregiver by SMS. The caregiver, using the app installed in his smartphone, analyses the message, does some preliminary pre-defined first aid and sends outcomes to a professional caregiver (doctor) for professional advice. The informal caregiver app also serves to further transmit, immediately or periodically, collected data to a central server through the Internet.

In Fig. 4, which shows the interconnection platform and services management, the communication is mediated by ThingSpeak ${ }^{4}$, an open source IoT application and API to store and retrieve data from things using the HTTP protocol over the Internet or via a Local Area Network. ThingSpeak enables the creation of sensor logging applications, location tracking applications, and a social network of things with status updates. The

${ }^{4}$ https://thingspeak.com/ 
doctor, using a Web or a mobile app, can access the patient's data that have been previously collected from the iHealthBag device and later transferred by the informal caregiver app on ThingSpeak.

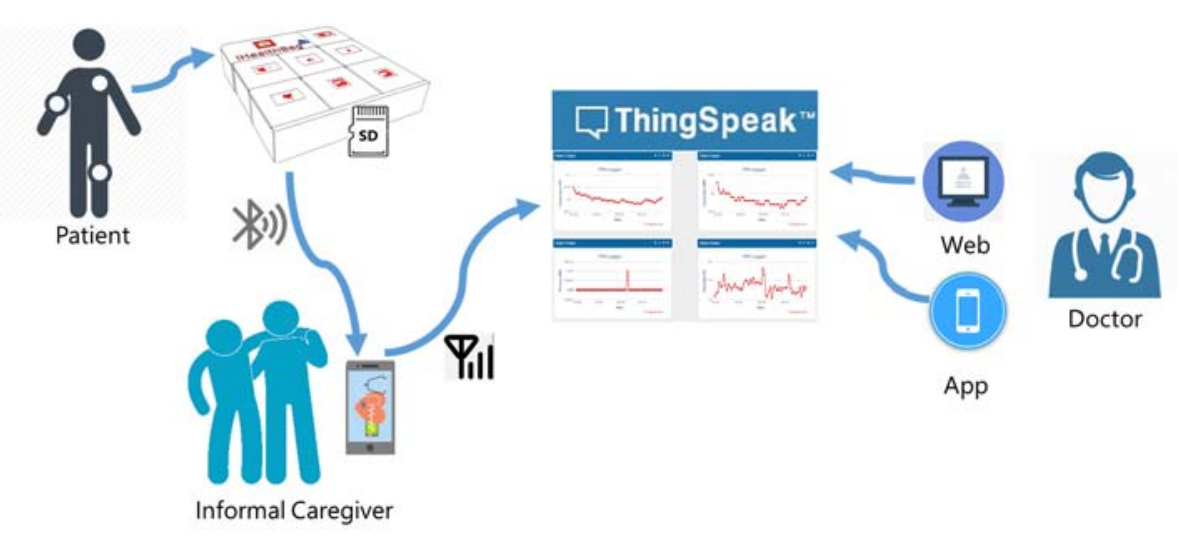

Fig. 4. Diagram representing the different components and users of the iHealthBag overall system.

\section{Discussion and Conclusion}

In this paper we have discussed the design concept of an IoT system aiming at giving access to good health care in rural villages. Many possibilities are offered by IoT technology for addressing such an issue, but we had to take in consideration the many constraints and limitations of the specific context. Thus, in order to propose a system that can be actually adopted in the considered context, we had to manage a trade-off between effectiveness and efficiency of the designed solution and the specific context requirements. We are aware that further issues are very significant in the AAL domain, for example, sensor reliability and data security. Still we think that our project can represent a first valid product to satisfy the requirements that would make it accessible in the considered context, namely rural villages.

The next step we are going to perform is to validate our design concept by involving a larger number of stakeholders, including patients, informal caregivers and doctors. Then, we want to implement a fully operational prototype of the overall system to be tested in a longitudinal study, by distributing the iHealthBag device to a sample of end users. By exploiting our experience on approaches that allow people that are not expert of technology to easily manage mobile devices [19] and smart objects [10,20], and to configure their own applications (e.g., see [21,22]), we are now working at the implementation of the app for the informal caregiver's mobile device as well as both the web app and the mobile app to be provided to the professional caregivers.

Acknowledgments. This work is partially funded by the Apulia Region through the project GLOBALDOC (CUP H96J17000160002, approved with A.D. n. 9 on 18/01/2017) and by the Italian Ministry of Education, University and Research (MIUR) 
through PON Ricerca e Innovazione 2014-2020 - Asse I "Investimenti in capitale umano" - Azione I.1 "Dottorati Innovativi con caratterizzazione industriale" (CUP H92H18000210006 and H92H18000200006 approved with D.R.n.991 on 29/03/2018 of University of Bari Aldo Moro).

\section{References}

1. Fiske, A., Wetherell, J.L., Gatz, M.: Depression in Older Adults. Annual Review of Clinical Psychology 5(1), pp. 363-389 (2009)

2. Huang, Y.-P., Huang, C.-Y., Liu, S.-I.: Hybrid intelligent methods for arrhythmia detection and geriatric depression diagnosis. Applied Soft Computing 14, pp. 38-46 (2014)

3. Sisinni, E., Saifullah, A., Han, S., Jennehag, U., Gidlund, M.: Industrial Internet of Things: Challenges, Opportunities, and Directions. IEEE Transactions on Industrial Informatics, pp. 1-1 (2018)

4. Gubbi, J., Buyya, R., Marusic, S., Palaniswami, M.: Internet of Things (IoT): A vision, architectural elements, and future directions. Future Generation Computer Systems 29(7), pp. $1645-1660$ (2013)

5. Risteska Stojkoska, B.L., Trivodaliev, K.V.: A review of Internet of Things for smart home: Challenges and solutions. Journal of Cleaner Production 140, pp. 1454-1464 (2017)

6. Ryu, M., Kim, J., Yun, J.: Integrated Semantics Service Platform for the Internet of Things: A Case Study of a Smart Office. Sensors 15(1), pp. 2137-2160 (2015)

7. Amandeep, Bhattacharjee, A., Das, P., Basu, D., Roy, S., Ghosh, S., Saha, S., Pain, S., Dey, S., Rana, T.K.: Smart farming using IOT. In: IEEE Annual Information Technology, Electronics and Mobile Communication Conference (IEMCON '17). pp. 278-280 (2017)

8. Kaluvala, N.S., Forman, A.: Smart Grid. Int. J. E-Polit. 4(2), pp. 39-47 (2013)

9. Gascó-Hernandez, M.: Building a smart city: lessons from Barcelona. Communications of the ACM 61(4), pp. 50-57 (2018)

10. Ardito, C., Buono, P., Desolda, G., Matera, M.: From smart objects to smart experiences: An end-user development approach. International Journal of Human-Computer Studies 114, pp. 51-68 (2018)

11. Zancanaro, M., Not, E., Petrelli, D., Marshall, M., van Dijk, T., Risseeuw, M., van Dijk, D., Venturini, A., Cavada, D., Kubitza, T.: Recipes for tangible and embodied visit experiences. In: Museums and the Web conference (MW '15) (2015)

12. Varshney, U.: Pervasive Healthcare and Wireless Health Monitoring. Mobile Networks and Applications 12(2), pp. 113-127 (2007)

13. Dishman, E.: Inventing wellness systems for aging in place. Computer 37(5), pp. 34-41 (2004)

14. Park, K., Pak, J.: Implementation of a Handheld Compute Engine for Personal Health Devices. International Journal of Smart Home 6(2), pp. 59-64 (2012)

15. Ranjan, R., Varma, S.: Object-oriented Design for Wireless Sensor Network assisted Global Patient Care Monitoring System. International Journal of Computer Applications 45(2), pp. 8-15 (2012) 
16. Wu, M.Y., Huang, W.Y.: Health care platform with safety monitoring for long-term care institutions. In: International Conference on Networked Computing and Advanced Information Management (NCM '11). pp. 313-317 (2011)

17. Buono, P., Cassano, F., Legretto, A., Piccinno, A.: A Modular Pill Dispenser Supporting Therapies at Home. In: International Workshop on Engineering the Web of Things (EnWoT '18) (2018)

18. Ardito, C., Buono, P., Costabile, M.F., Desolda, G., Lanzilotti, R., Piccinno, A., Matera, M.: Enabling end users to define the behaviour of smart objects in AAL scenarios In: Forum Italiano Ambient Assisted Living (ForITAAL '18) (2018)

19. Desolda, G., Ardito, C., Jetter, H.-C., Lanzilotti, R.: Exploring spatially-aware cross-device interaction techniques for mobile collaborative sensemaking. International Journal of Human-Computer Studies 122, pp. 1-20 (2019)

20. Desolda, G., Ardito, C., Matera, M.: Empowering End Users to Customize their Smart Environments: Model, Composition Paradigms, and Domain-Specific Tools. ACM Trans. Comput.-Hum. Interact. 24(2), pp. Article N. 12 - 11-52 (2017)

21. Ardito, C., Costabile, M.F., Desolda, G., Latzina, M., Matera, M.: Making mashups actionable through elastic design principles. In: Díaz, P., Pipek, V., Ardito, C., Jensen, C., Aedo, I., Boden, A. (eds.), End-User Development - IS-EUD 2015. Vol. LNCS 9083, pp. 236-241. Springer, Berlin Heidelberg (2015)

22. Desolda, G., Ardito, C., Matera, M.: EFESTO: A Platform for the End-User Development of Interactive Workspaces for Data Exploration. In: Daniel, F., Pautasso, C. (eds.), Rapid Mashup Development Tools: First International Rapid Mashup Challenge, RMC 2015, Rotterdam, The Netherlands, June 23, 2015, Revised Selected Papers, pp. 63-81. Springer International Publishing, Cham (2016) 